\title{
A PRELIMINARY REPORT ON OOCYTE DONATION EXTENDING REPRODUCTIVE POTENTIAL TO WOMEN OVER 40
}

\author{
Mark V. Sauer, M.D., Righard J. Paulson, M.D., and Rogerio A. Lobo, M.D.
}

\begin{abstract}
Background. Fertility in women 40 years of age or older is decreased, and in those with ovarian failure it is thought to be irrevocably lost. The donation of oocytes to young $(<35$ years old) women with ovarian failure has allowed many considered infertile a chance to become pregnant. In these women gonadal hormone replacement results in an endometrium receptive to implantation. It is not known whether the endometrial response to such replacement is decreased in women over the age of 40 .
\end{abstract}

Methods. To test the efficacy of oocyte donation to older women, we enrolled seven women 40 to 44 years old with ovarian failure in a trial of hormone replacement and embryo transfer, using oocytes obtained from women undergoing ovarian hyperstimulation solely for gamete donation.

Results. Seven stimulated cycles in the donors that were synchronized with nine cycles in the recipients resulted in eight embryo transfers. Five viable pregnancies

$\mathrm{F}$ ERTILITY in women over the age of 40 is generally acknowledged to be decreased. In analyzing a large series of women, Leridon suggested that fecundity in women actually begins to decline by 30 years of age. ${ }^{1}$ Fertility rates peak by the age of 25 and fall throughout the remainder of reproductive life. Although many in vitro fertilization programs accept women over 40 , the pregnancy rates in such women have been reported to be decreased, ${ }^{2}$ and the rates of miscarriage may be increased. ${ }^{3}$ Fertility is similarly diminished in older women undergoing donor insemination. ${ }^{4}$ In them, the cumulative success rates for pregnancy fall after they reach 30 years of age and decline dramatically after age $35 .{ }^{5}$

As many as 10 percent of women have ovarian failure by 40 years of age, ${ }^{1}$ and until recently these women were considered to be hopelessly infertile. With aging, diminished fertility is believed to occur principally as a result of oocyte senescence, but it could also be due to resistance to implantation of the embryo in the endometrium. ${ }^{2}$ After implantation, the loss of pregnancy is also increased in women over 40 , presumably as a result of a combination of detrimental changes in aging oocytes and the endometrium.

We previously reported the establishment of a program involving the nonanonymous donation of oocytes. ${ }^{6}$ Initially, younger women provided oocytes to women under the age of 40 with ovarian failure. The donors were fertile women who underwent controlled ovarian hyperstimulation and transvaginal, ultrasound-directed aspiration of oocytes for the sole purpose of donation. Thirteen cycles were initiated,

From the Department of Obstetrics and Gynecology, Division of Reproductive Endocrinology, University of Southern California, Los Angeles. Address reprint requests to Dr. Sauer at Women's Hospital, 1240 N. Mission Rd. , Rm. L946, Los Angeles, CA 90033. were established, one with twins. A sixth pregnancy ended in miscarriage. Five normal infants were delivered by cesarean section, and one stillborn infant was delivered vaginally. The outcomes were compared with those in women under the age of 40 with ovarian failure who were also participating in our donor-oocyte program and in infertile ovulating women 40 or older who were undergoing standard in vitro fertilization. No significant differences in rates of implantation or ongoing pregnancy were noted in older women as compared with younger women receiving donated embryos. These rates, however, were higher than the rates in the infertile ovulating women of similar age who were undergoing standard in vitro fertilization.

Conclusions. These preliminary results suggest that the endometrium retains its ability to respond to gonadal steroids and provides a receptive environment for embryo implantation and gestation even in older women. ( $\mathrm{N}$ Engl J Med 1990; 323:1157-60.)

resulting in 11 embryo transfers and 6 ongoing pregnancies. Encouraged by our preliminary experience, we expanded the protocol to include women over the age of 40 with ovarian failure who were otherwise in good physical and psychological health. In this article we report the results of our initial trials of oocyte donation in these older patients. Since the oocytes were donated by a group of young, fertile women, we could determine whether age alone affects the receptivity of the uterus to embryo implantation.

\section{Methods}

Between February 1, 1989, and September 1, 1989, seven infertile couples consented to undergo at least one cycle of in vitro fertilization with donated oocytes. All the recipients were married women 40 to 44 years old who had previously been found to have primary ovarian failure. In all the women, the ovarian failure was considered to be idiopathic and had occurred at least two years before enrollment in our study. All the women had serum follicle-stimulating hormone values above 100 IU per liter and estradiol levels below 90 pmol per liter. There was no attempt to induce ovulation in these women because of the longstanding and severe nature of their ovarian failure, as demonstrated by their very high levels of folliclestimulating hormone.

None of the women had any major medical problem. The couples and donors were also interviewed by a psychologist before study entry and deemed acceptable candidates for embryo donation. The couples were advised of the possible increased obstetrical risks in pregnant women over the age of $40 .^{7}$ Individual consent forms were signed by the donors, the recipients, and the recipients' husbands. The protocol for embryo donation had been reviewed and approved by the institutional review board of the California Medical Center, Los Angeles.

The women with ovarian failure were treated with oral micronized estradiol and intramuscular progesterone as previously described (Fig. 1). ${ }^{8}$ The medications were administered in divided doses and taken twice daily, except that estradiol was taken in three doses on the days when the dose was $6 \mathrm{mg}$. After this regimen of hormones, a histologically normal endometrial biopsy specimen was obtained from each recipient on day 26 of a simulated replacement cycle within two months of the embryo-transfer cycle.

All the women receiving embryos had a normal endometrial cav- 


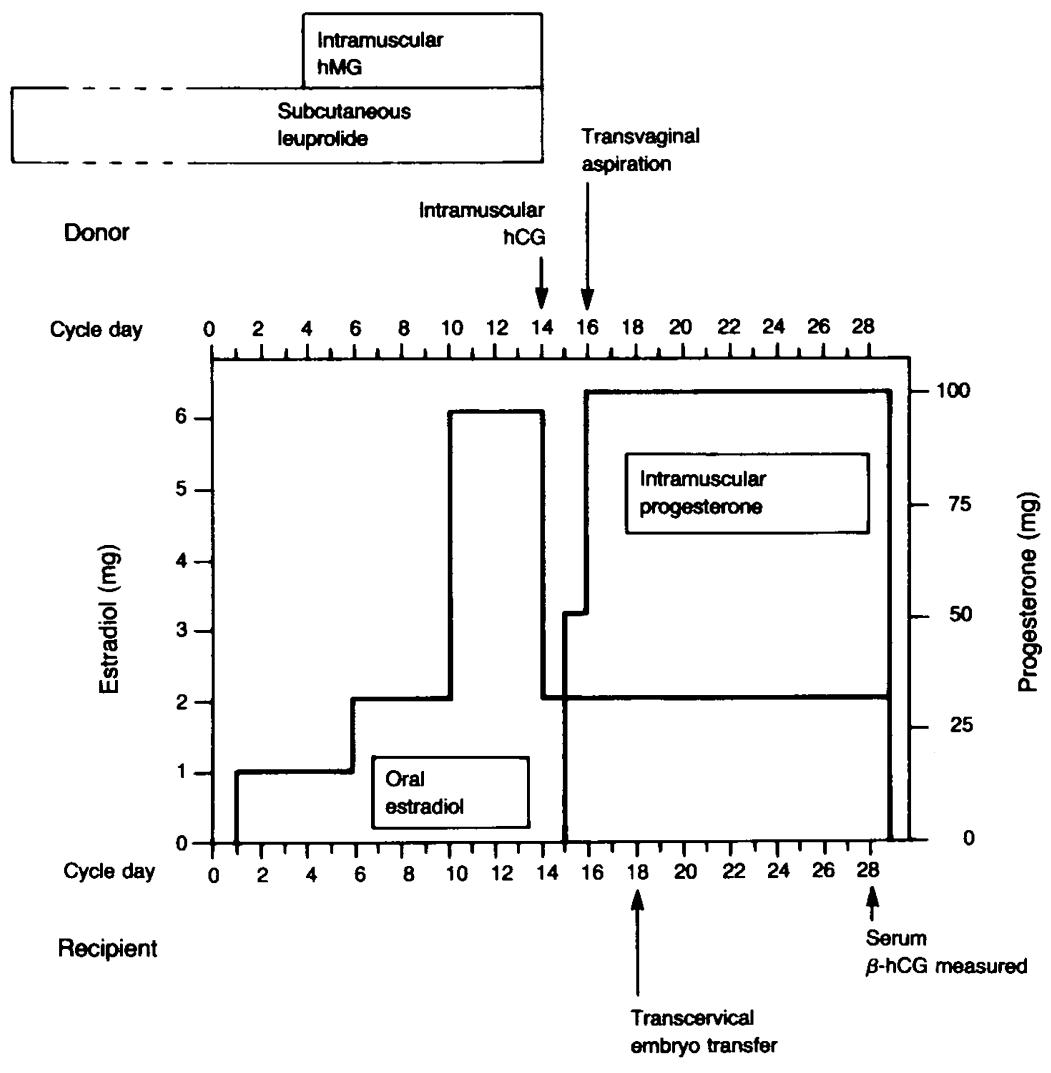

Figure 1. Regimens of Ovarian Stimulation and Hormone Replacement Used to Synchronize the Development of Ovarian Follicles in the Oocyte Donor and the Endometrial Cycle in the Recipient.

hMG denotes human menopausal gonadotropin, hCG human chorionic gonadotropin, and $\beta$-hCG the beta subunit of hCG.

ity, as determined by hysterosalpingography, as well as a normal mammogram, electrocardiogram, and fasting blood glucose concentration, and a negative serologic test for human immunodeficiency virus (HIV) infection. Blood type and Rh status were also determined. The husbands of the recipients also had negative tests for HIV infection and negative semen cultures.

The oocytes were provided by three fertile women 31 to 34 years old who were paid $\$ 1,500$ per cycle by the recipients. Each had regular ovulatory cycles, no gynecologic abnormalities, and no major medical or psychological illness. Because of the large number of oocytes retrieved from one of these donors during a previous stimulated cycle ${ }^{9}$ her ovarian hyperstimulation cycle was synchronized on two occasions with the cycles of two recipients. The donors underwent controlled ovarian hyperstimulation with use of subcutaneous leuprolide acetate (1 mg daily) and intramuscular human menopausal gonadotropin (three ampules daily) as previously described. ${ }^{6}$ Leuprolide was begun during the mid-luteal phase of the donor's menstrual cycle. Human menopausal gonadotropin was begun once the donor's serum estradiol level fell below 90 pmol per liter. Ultrasound-directed transvaginal aspiration of follicles was performed under intravenous sedation 36 hours after the intramuscular administration of human chorionic gonadotropin. The recovery of oocytes, fertilization, embryo culture, and subsequent transfer of the embryo were performed by standard methods of in vitro fertilization currently used in our program and described previously. ${ }^{6}$ All transfers of embryos were performed transcervically, without anesthesia.

The recipients began receiving estradiol replacement three days before the donor began receiving human menopausal gonadotropin and then started progesterone replacement on the morning the donor received human chorionic gonadotropin (Fig. 1). The day progesterone replacement began was arbitrarily designated as day 15 of the simulated cycle. The embryos were transferred on day 18 of the cycle. Pregnancies were confirmed by the measurement of serum levels of the beta subunit of human chorionic gonadotropin and by transvaginal ultrasonography. Individual implantations were verified by the presence of a distinct gestational sac on ultrasonography. Clinical pregnancies were confirmed by visible heartbeats within the gestational sacs at least eight weeks after the transfer of the embryo. All the women who became pregnant continued to receive $2 \mathrm{mg}$ of estradiol orally and $100 \mathrm{mg}$ of progesterone intramuscularly in daily divided doses for an additional 100 days.

The outcomes in the group of recipients were compared with those in two other groups in our in vitro fertilization program. The first group $(n=9)$ was composed of all the women under 40 with primary ovarian failure who had participated in the donoroocyte program since its inception. The mean age in this group was 32 years (range, 27 to 39$)$. The second group $(n=22)$ consisted of all ovulating but infertile women 40 or older (mean, 41 ) who participated in our standard program of in vitro fertilization from January 1987 through August 1989. Women undergoing ovarian hyperstimulation received either human menopausal gonadotropin (initial dose, $225 \mathrm{IU}$ a day) followed by human chorionic gonadotropin (10,000 IU) when the follicles were mature, or leuprolide acetate and then human menopausal gonadotropin (225 IU a day) followed by human chorionic gonadotropin $(10,000 \mathrm{IU})$ when the follicles were mature. We performed transvaginal aspiration, gamete and embryo culture, and transcervical transfer of the embryo, using the same gamete laboratory and personnel as previously described. ${ }^{6}$

The numbers of oocytes obtained, fertilized, and transferred and the rates of implantation and pregnancy were compared in the three groups. The differences between groups were analyzed with either the nonparametric Mann-Whitney test or Fisher's exact test, as appropriate. A $\mathbf{P}$ value of less than 0.05 was considered to indicate significance.

\section{Results}

Table 1 shows the outcomes of each of the cycles in the seven women 40 or older who received embryos. Five women underwent one cycle and two underwent two cycles. Six pregnancies resulted from eight transfers of embryos to these women. In one instance no transfer was performed because there was no fertilized oocyte. A total of 28 embryos were transferred during the eight transfers (mean $[ \pm \mathrm{SD}], 3.5 \pm 1.1$ embryos per transfer). Ten implantations resulted, for an implantation rate of 36 percent. Six of the seven women became pregnant. One pregnancy ended in a miscarriage after nine weeks of gestation. In one woman the pregnancy progressed normally, but the fetus died in the uterus after 40 weeks of gestation; no cause of stillbirth was apparent. The pregnancies in the other four women were normal, and they all delivered normal infants. 
Table 1. Results of the Transfer of Fertilized Donor Ova in Women $\mathbf{4 0}$ or Older with Ovarian Failure.

\begin{tabular}{|c|c|c|c|c|c|}
\hline $\begin{array}{l}\text { Patient } \\
\text { No. }\end{array}$ & $\begin{array}{l}\text { AGE } \\
\text { (YR) }\end{array}$ & $\begin{array}{l}\text { No. FerTILIZED*/ } \\
\text { Total. No. }\end{array}$ & $\begin{array}{l}\text { No. TRANS- } \\
\text { fERRED }\end{array}$ & $\begin{array}{l}\text { No. IM- } \\
\text { PLANTED } \dagger\end{array}$ & OUTCOME $\ddagger$ \\
\hline 1 & 41 & $4 / 6$ & 4 & 2 & $\begin{array}{l}\text { Singleton pregnancy; } \\
\text { CS week } 37\end{array}$ \\
\hline 2 & 40 & $6 / 10$ & 6 & 1 & $\begin{array}{l}\text { Singleton pregnancy; } \\
\text { CS week } 38\end{array}$ \\
\hline 3 & 44 & $1 / 21$ & 1 & 1 & $\begin{array}{l}\text { Singleton pregnancy } \\
\text { CS week } 40\end{array}$ \\
\hline 4 & 41 & $\begin{array}{c}16 / 20 \\
5 / 6\end{array}$ & $\begin{array}{l}5 \\
5\end{array}$ & $\begin{array}{l}0 \\
0\end{array}$ & $\begin{array}{l}\text { Not pregnant } \\
\text { Not pregnant }\end{array}$ \\
\hline 5 & 40 & $\begin{array}{l}0 / 10 \\
2 / 10\end{array}$ & $\begin{array}{l}0 \\
2\end{array}$ & $\begin{array}{l}0 \\
1\end{array}$ & $\begin{array}{l}\text { No fertilization } \\
\text { SAB week } 9\end{array}$ \\
\hline 6 & 41 & $3 / 8$ & 3 & 2 & $\begin{array}{l}\text { Singleton pregnancy } \\
\text { NSVD, death of } \\
\text { fetus week } 40\end{array}$ \\
\hline 7 & 43 & $3 / 6$ & 2 & 2 & $\begin{array}{l}\text { Twin pregnancy; } \\
\text { CS week } 38\end{array}$ \\
\hline
\end{tabular}

*The number of donor ova fertilized by sperm from the recipient's spouse.

tThe number of gestational sacs visualized by transvaginal ultrasonography four weeks after transfer.

‡CS denotes cesarean section, SAB spontaneous clinical abortion, and NSVD normal spontaneous vaginal delivery.

Table 2 shows the results in the patients with ovarian failure who were 40 or older as compared with those in the younger patients with ovarian failure in our oocyte-donation program and those in the infertile ovulating women 40 or older who were undergoing ovarian hyperstimulation and oocyte aspiration in our in vitro fertilization program. There were no significant differences in outcome in the older as compared with the younger women with ovarian failure. Among the older women who underwent standard in vitro fertilization and embryo transfer, significantly fewer oocytes were retrieved, fewer embryos were transferred, and the rates of implantation and pregnancy were lower than in either the younger or older women who received donated embryos.

The overall rate of oocyte fertilization in the older women with ovarian failure was 41 percent ( 40 of the 97 oocytes retrieved). This was lower than the overall rate of fertilization (62 percent) among couples without male-factor infertility in our general in vitro fertilization program in 1988 (data not shown; $\mathrm{P}<0.05)$. The husbands of 2 of the 7 women in the study group, as well as those of 6 of the 22 infertile ovulating women who were 40 or older, were suspected of being infertile on the basis of abnormalities in their screening laboratory examinations $(0$ percent score on the hamster penetration assay). The rate of fertilization was lower in both groups than in the group of women with ovarian failure who were under 40 , none of whose husbands had such abnormalities. The poor rates of fertilization in two older. women (Patients 3 and 5) contributed greatly to the low overall rate. Despite poor fertilization rates in these two $(0$ of 10 and 2 of 10 oocytes in Patient 5, and 1 of 21 oocytes in Patient 3), when fertilization occurred the zygotes cleaved, implanted, and continued to develop in both cases, and the pregnancy was successful in Patient 3.

\section{Discussion}

Ovarian failure occurs in most women in the United States between the ages of 48 and 51.,10 Many women are either menopausal or perimenopausal by the time they reach their early forties. For women who delay childbearing until late in their reproductive lives, the likelihood of starting a family is decreased. For those with ovarian failure there are few options for pregnancy.

We have previously demonstrated that high rates of pregnancy can be achieved in women with premature ovarian failure if multiple embryos are transferred to them after hormonal stimulation of the endometrium. ${ }^{6}$ The rate of pregnancy in such women is approximately three times that reported by the national registry for infertile ovulating women who undergo standard in vitro fertilization and embryo transfer. ${ }^{11}$ We believe that the increased rate of embryo implantation results both from the receptive uterine environment artificially created in these recipients and from the transfer of multiple embryos from oocytes obtained from young, fertile donors.

The endometrium of women with ovarian failure can be made histologically normal with a combination of oral micronized estradiol and intramuscular progesterone. ${ }^{8}$ Endometrial-biopsy specimens from women receiving this hormone replacement regimen (both those under and those over 40 ) are histologically indistinguishable from normal endometrium. We therefore believed that in older women with ovarian failure a pregnancy rate similar to that of younger women with

Table 2. Results of the Transfer of Fertilized Donor Ova in Women 40 or Older with Ovarian Failure, as Compared with the Results in Women under 40 with Ovarian Failure and Those of Women over 40 Undergoing Standard in Vitro Fertilization (IVF) and Embryo Transfer.*

\begin{tabular}{|c|c|c|c|}
\hline & $\begin{array}{c}\text { WOMEN }>40 \\
\text { WITH DONOR IVF }\end{array}$ & $\begin{array}{c}\text { WOMEN }<40 \\
\text { WITH DONOR IVF }\end{array}$ & $\begin{array}{c}\text { WOMEN }>40 \\
\text { WITH STANDARD IVF }\end{array}$ \\
\hline No. of recipients & 7 & 9 & 22 \\
\hline Recipient cycles & 9 & 14 & 31 \\
\hline Oocytes per recipient & $10.8 \pm 5.8$ & $13.6 \pm 6.8$ & $5.8 \pm 3.5 \dagger$ \\
\hline $\begin{array}{l}\text { Fertilized oocytes/oocytes retrieved } \\
\text { (fertilization rate) }\end{array}$ & $40 / 97(41)$ & $125 / 191(65) \dagger$ & $78 / 199(39)$ \\
\hline Transfer cycles & 8 & 14 & 26 \\
\hline Embryos transferred per cycle & $3.5 \pm 1.1$ & $4.8 \pm 0.7$ & $2.6 \pm 1.6 \ddagger$ \\
\hline Implantations per transferred embryo & $10 / 28(36)$ & $18 / 67 \quad(27)$ & $6 / 83(7) \dagger$ \\
\hline $\begin{array}{l}\text { Clinical pregnancies per transfer } \\
\text { attempt }\end{array}$ & $6 / 8 \quad(75)$ & $7 / 14 \quad(50)$ & $4 / 25 \quad(16) \dagger$ \\
\hline $\begin{array}{l}\text { Ongoing pregnancies or live births } \\
\text { per transfer attempt }\end{array}$ & $5 / 8 \quad(62)$ & $7 / 14 \quad(50)$ & $2 / 25 \quad(8) \dagger$ \\
\hline
\end{tabular}

*Plus-minus values are means $\pm \mathrm{SD}$. Values in parentheses are percentages.

$+\mathrm{P}<0.05$ for the comparison with the other groups.

$\pm \mathrm{P}<0.05$ only for the comparison with recipients $<40$ years old; $\mathrm{P}$ not significant for the comparison with recipients 40 or 
the same problem was possible, provided that embryos of similar quality were transferred. Indeed, the rate of implantation (36 percent) in this older group was not different from that previously reported by us in women under the age of 35 (35 percent). ${ }^{12}$

The incidence of genetic anomalies in the offspring of women undergoing in vitro fertilization and embryo transfer is not different from that in the population at large. ${ }^{11}$ Whether the incidence of such anomalies in this older group of women with ovarian failure will resemble that of the younger donors who provide the gametes or that of their own age group is a matter of speculation. The increased incidence of anomalies in the offspring of older women in general may be due to either the older gametes or lack of rejection of abnormal conceptuses by the endometrium of older women. Similarly, the rate of miscarriage in these older women is not yet known, but our results suggest that it is not different from that of younger women in the general population.

The obstetrical risk to the women in this study is unknown. However, infant mortality in women 40 or older is significantly increased ( 12 deaths per 1000 live births at age 40 vs. 9 per 1000 at age 30). ${ }^{13}$ Maternal mortality rates are also higher (80 per 100,000 live births at age 40 vs. 20 per 100,000 at age 30$).{ }^{14}$ If highrisk obstetrical care is provided, the rate of obstetrical complications may not be appreciably higher than that in younger women. ${ }^{15}$ Careful screening of patients will further lessen complications if those with known high-risk factors, such as hypertension, diabetes mellitus, or thromboembolic disease, are excluded from participation. Since the risks are currently unknown, all women should be carefully screened and counseled regarding pregnancy before oocyte donation is attempted.

Oocyte donation provides older women with ovarian failure a reasonable chance at achieving pregnancy. To those with existing ovarian function, conventional in vitro fertilization and embryo transfer may still be offered, although the success of pregnancy is probably reduced and at best results in a live-born child in less than 10 percent of the initiated cycles. ${ }^{2}$ A recent report suggested that the creation of a functionally agonadal state by means of pituitary downregulation with a gonadotropin-releasing hormone agonist followed by ovarian hormone replacement allows the cycles of selected women with ovarian function to be synchronized with those of an oocyte donor. ${ }^{16}$ Such a protocol may be applicable to women whose ovarian failure is intermittent, as is often the case in perimenopausal women.

In this small series, the rates of pregnancy with donated oocytes in women with ovarian failure who were 40 or older approximated that in younger women in the same program, demonstrating that receptivity to embryo implantation can be maintained if not enhanced by hormone replacement therapy in such older women. The probability of randomly achieving pregnancy in women with ovarian failure in this age group with any other existing method is extremely low. Since women in this age group with functioning ovaries are considered appropriate candidates for infertility treatment, it seems appropriate that those without ovarian function should also be allowed a chance to experience pregnancy if they desire it.

\section{REFERENCES}

1. Leridon H. Human fertility: the basic components. Chicago: University of Chicago Press, 1977

2. Edwards RG, Fishel SB, Cohen J, et al. Factors influencing the success of in vitro fertilization for alleviating human infertility. J In Vitro Fert Embryo Transf 1984; 1:3-23.

3. Romeu A, Muasher SJ, Acosta AA, et al. Results of in vitro fertilization attempts in women 40 years of age and older: the Norfolk experience. Fertil Steril 1987; 47:130-6.

4. Fédération CECOS, Schwartz D, Mayaux MJ. Female fecundity as a function of age: results of artificial insemination in 2193 nulliparous women with azoospermic husbands. N Engl J Med 1982; 306:404-6.

5. Virro MR, Shewchuk AB. Pregnancy outcome in 242 conceptions after artificial insemination with donor sperm and effects of maternal age on the prognosis for successful pregnancy. Am J Obstet Gynecol 1984; 148:51824.

6. Sauer MV, Paulson RJ, Macaso TM, Francis-Hernandez M, Lobo RA Establishment of a nonanonymous donor oocyte program: preliminary experience at the University of Southern California. Fertil Steril 1989; 52:433-6.

7. Kajanoja P, Widholm O. Pregnancy and delivery in women aged 40 and over. Obstet Gynecol 1978; 51:47-51.

8. Sauer MV, Paulson RJ. Oocyte donation to women with ovarian failure. Contemp Obstet Gynecol 1989; 34:125-35.

9. Sauer MV, Paulson RJ, Lobo RA. Simultaneous establishment of pregnancies in two ovarian failure patients using one oocyte donor. Fertil Steril $1989 ; 52: 1072-3$

10. Mishell DR Jr. Menopause. In: Droegemuellar W, Herbst AL, Mishell DR, Stenchever MA, eds. Comprehensive gynecology. St. Louis: C.V. Mosby, 1987:1082-107.

11. In vitro fertilization/embryo transfer in the United States: 1987 results from the National IVF-ET Registry. Fertil Steril 1985; 51:13-9.

12. Paulson RJ, Sauer MV, Lobo RA. Embryo implantation following human in vitro fertilization: importance of endometrial receptivity. Fertil Steril 1990; 53:870-4.

13. Friede A, Baldwin W, Rhodes PH, Buehler JW, Strauss LT. Older maternal age and infant mortality in the United States. Obstet Gynecol 1988; 72:1527 .

14. Rochat RW, Koonin LM, Atrash HK, Jewett JF, Maternal Mortality Collaborative. Maternal mortality in the United States: report from the Maternal Mortality Collaborative. Obstet Gynecol 1988; 72:91-7.

15. Kirz DS, Dorchester W, Freeman RK. Advanced maternal age: the mature gravida. Am J Obstet Gynecol 1985; 152:7-12.

16. Meldrum DR, Wisot A, Hamilton F, Gutlay-Yeo AL, Marr B, Huynh D. Artificial agonadism and hormone replacement for oocyte donation. Fertil Steril 1989; 52:509-11. 